\title{
Urban Health Insurance Reform in China
}

\author{
Hai-Chang XIN ${ }^{1, a}$, Jonathan AMSBARY2,b, ${ }^{,}$, Larry POWELL ${ }^{3, c}$ \\ ${ }^{1}$ Statistician with the Department of Health Care Organization \& Policy, University of Alabama - \\ Birmingham, Birmingham AL USA 35294 \\ ${ }^{2}$ Associate professor, Communication Studies, UAB, Birmingham AL USA 35294 \\ ${ }^{3}$ Professor, Communication Studies, UAB, Birmingham AL USA 35294 \\ ahxin@uab.edu, bamsbary@uab.edu, 'Ipowell@uab.edu \\ *Corresponding author
}

\begin{abstract}
Keywords: Health care, Health care systems, Chinese health care, Government health insurance, Chinese health insurance, Chinese Government Insurance Scheme, Chinese Labor Insurance Scheme.
\end{abstract}

\begin{abstract}
The healthcare system for urban employees in China was established in the early 1950s. The first stage (1950s-1980) mainly consisted of two parts, the Government Insurance Scheme (GIS) and the Labor Insurance Scheme (LIS). The GIS and LIS achieved remarkable success with limited resources, contributed to economic development and social stability, and won high praise from the World Health Organization. The second stage (1980s to date) aimed at controlling costs and expanded the risk pool to the city level, but experienced several problems, was not well received by the public, and drew negative government evaluations. This study examines underlying reasons and offers recommendations for improvement.
\end{abstract}

\section{Stage I: 1950s-1980}

The health insurance system for China's urban workers was first established in the early 1950s and consisted of two parts: the Government Insurance Scheme (GIS), which covered government employees, retirees, disabled veterans, and university teachers, staff and students; and the Labor Insurance Scheme (LIS), which covered state enterprise employees, retirees and their dependents. Only state-owned enterprises (SOE, enterprises owned and managed by central or provincial governments) with more than 100 employees were required to participate in the LIS; smaller state enterprises and industries owned by local governments participated on a voluntary basis. Both the GIS and LIS provided "longitudinal" insurance, i.e., individual government agency and SOE only insured their own employees and there was no linkage and reciprocity across them at the community level. Both approaches were financed by the government.

The GIS and LIS provided comprehensive health care benefits. Beneficiaries received free outpatient, inpatient services and prescription drugs, which were often called "Free Medical Care." Dependents of the LIS beneficiaries were provided with a 50 percent coinsurance for their medical service and drugs. Small collectively owned enterprises enrollees enjoyed similar benefits to those of rural workers. Each collective unit ran its own insurance programs. Their employees were usually covered but not necessarily their dependents. Often the employees had to pay for their medical expenses first and to obtain reimbursement later.

China developed a three-tiered organization for the delivery of health care. The three-tier hospitals had similar functions but were different in scale and "target" groups. In Shanghai's case, the three levels were the municipal, district and street level. Each level covered a defined beneficiary population, and lower-level hospitals could refer patients to higher ones when it was unable to treat them due to a lack of necessary skills and/or equipment. In addition, most large work units had their own hospitals, 
and medium and small firms/institutions had their own clinics or health care stations. This system referred patient to the most appropriate setting thereby ensured efficient health resource allocation.

\section{Performance}

The performance of this Chinese health insurance system turned out to be a surprising success. The health status of the Chinese people rose dramatically during that period, mortality and morbidity rates were greatly reduced, many infectious diseases were effectively controlled, some sexual transmitted diseases were even eradicated, infant mortality fell from 200 to 34 per 1000 live births, and life expectancy increased from about 35 to 68 years [1]. The health improvements in China resulted in an interesting comparison reported by the New York Times [2]:

In Shanghai, 10.9 infants out of 1,000 die before their first birthday, while in New York City infant mortality is 13.3 per 1,000 live births. And life expectancy at birth in Shanghai is now 75.5 years, compared to a life expectancy in New York City of about 73 years for whites and 70 years for nonwhites as of 1980, the last year for which data are available.

By the beginning of the 1980s, China was undergoing the epidemiologic transition seen in Western countries: infectious diseases were giving way to chronic diseases (e.g., heart disease, cancer, and stroke) as leading causes of illness and death.

\section{Factors Affecting Success}

The underlying reasons for this success were complex, in a word, the health insurance system as well as delivery system matched China's social economic situation and population's health status and medical need perfectly. In 1950, the newly established government faced a situation racked by poverty and disease after long-term wars, malnutrition and endemic disease, and the industrial base was in ruins [3]. In politics, the government adopted systematically planned and strongly enforced organizational principles. Also, the government believed in health care as welfare in socialism system and thus a priority to which everyone should be entitled to receive. An ideology of equity for all citizens, and the near universal availability of adequate food, education, housing, jobs, and accessible and affordable health care services was advocated and widely accepted. Under these circumstances, China implemented four main principles guiding health care delivery: 1) medicine must serve the people; 2) priority should be given to prevention; 3) health education must be built on mass campaigns; and 4) The Chinese traditional medicine and the Western medicine should be integrated [4].

Thus several factors contributed to the program's success. The first was ideology congruency. Prior to implementation of the program, ordinary people were unable to improve their health conditions due to poverty. Once the government made a commitment to reduce diseases and ensure people's health, the government treated health and health care as a privilege in a socialist institution, leading to a model of egalitarian health care and universal coverage.

The second factor was operation feasibility. With a set of systematically planned and strongly enforced organizational principles, the government made full use of all its possible resources. Thus the government effectively set up the GIS and LIS and implemented health policies by via large scale health campaigns in nation-wide effort that supported the insurance system and quickly improved the public's health status.

The third factor was strategic appropriateness. Priorities were given to prevention and health education and implemented by mass campaigns. Massive immunization campaigns were carried out, brothels were closed, and campaigns against opium use were run. The Great Patriotic Health Campaigns mobilized the masses in tasks aimed at improving nutrition, sanitation, and water quality and attacking certain diseases [5]. These policies and cost-effective activities fit the societal conditions well: poverty, limited resources, lack of health infrastructure, low literacy rate and bad health behaviors. 
The fourth factor was organization appropriateness. The government integrated the health system within its industrial and agricultural systems - instead of a national health insurance or national health system. From 1950 to 1980, the GIS and LIS played an important role in providing China's urban population with health protection, given the fact that almost everyone in China belonged to a specific work-unit. In addition, the primary health care networks were also widely established in the community level, thereby ensuring people's maximum participation in the delivery and their easy universal access to the health services. The three-tiered delivery system effectively promoted the efficient allocation of health care resources between primary and tertiary care facilities.

\section{Stage II: 1980s to date}

The radical economic reform of the 1980s brought new challenges to China's health system. This reform process has experienced two major stages, the first from the early 1980s to 1991 and the second beginning in 1992 with city-wide pilot reforms [6].

During the first period (1980-1991), the primary objective was cost containment. Major reform measures included introduction of cost sharing both on the demand-side and supply-side. Before 1985, reforms were mostly focused on the demand side, such as the introduction of copayments. From 1985 to 1991 , the focus turned to the control of providers, especially economic incentives to hospitals, including pre-payment to hospitals according to the number of beneficiaries in their areas, and specifying a fixed fee for services. Moreover a limited list of pharmaceuticals was developed for which GIS beneficiaries would be reimbursed. In addition, effort was made to establish district or industry level risk pooling funds for catastrophic medical expenditures, which strengthened the financial ability of individual enterprises against catastrophic diseases.

After 1992 the focus of health reform shifted to the more fundamental problems - increasing the risk pool at the city level-along with the original goal of cost containment. With the pilot city reform experience from Shenzhen in Guangdong Province in 1992, Jiujiang in Jiangxi Province and Zhengjiang in Jiansu Province in 1995, China announced several major health sector reform initiatives in late 1990s. The major objectives of the current reform initiatives were to (a) establish a basic social insurance system for the urban worker; (b) control medical costs escalation; (c) and improve efficiency and quality [7].

In December of 1998, the Chinese government announced a major decision to establish a social insurance program for urban workers [8]. The new program added coverage to employees from private enterprises and smaller public enterprises. Self-employed and rural industry workers could buy into the program, but were not required to enroll. Workers' dependents were not covered. In essence, the urban health insurance system reforms in China intended to establish a social insurance system that would provide affordable health care benefits to urban workers by pooling risks at the city level.

1. Financing. The social insurance program is financed by premiums from employers who contribute 6 perccent of the employee's wage and employees who contributed 2 percent of their wage. Former employers shouldered the responsibility for paying for their retired workers' premium [9]. SOEs and government institutions are not required to participate in the program.

2. Benefit design. The social insurance program finances beneficiaries' health care services through a three-tier approach: individual medical savings accounts (MSAs); out-of-pocket spending by beneficiaries in the form of deductibles; and a social risk pooling fund (SRP). Among total contributions, 3.8 percent of the employee's wage goes into the MSA, and 4.2 percent of their wage goes into the SRP. In principle outpatient medical expenses are paid out of the MSAs until the funds have been depleted. In addition, enrollees have to pay outpatient expenses out-of-pocket. For inpatient hospital expenses, enrollees must first pay a deductible equal to 10 percent of their annual wage. That allows them to participate in the SRP with a coinsurance when expenses exceed the deductible (the rate of which is at the discretion of the local governments). The SRP has a ceiling up to four times the 
average wage of the workers in that city. To cover expenses above this ceiling, enrollees can use supplementary insurance plans, or those expenses must be paid out-of-pocket. Supplementary insurance coverage is only provided by the government for their employees, but other employers may purchase supplementary insurance for their employees. The employees can also purchase private supplementary insurance. Essential Drugs Lists and Essential Services Lists have also been developed in the intent of containing costs; these lists specify which drugs and services are covered (or not covered) by the program. The most restrictive of these lists are established by the central government; local governments can expand or reduce the items on the lists by up to 5 percent [10].

\section{Performance}

The current health insurance system reforms have mitigated some financial pressure on individual government agencies and the SOE and have also solved some cost problems. However, the system has also encountered problems. According to a report by the World Health Organization, in 2000 China ranked No.188 in "fairness of financial contribution" and No.144 in "overall health system performance" of all the 191 member countries. In 2005 the report from the Development Research Centre of the State Council (DRCSC), China's cabinet, claimed that the reform of China's medical and health system has been "basically unsuccessful", and there were "major" philosophical and practical problems with the reform, which was evidenced by several factors. First, there was a decline in both the fairness of medical services and the efficiency of investment in the sector. Second, the "business- and market-orientation" of the medical and health system was "absolutely wrong" and conflicted with the goal of public health. Third, There are obvious flaws with the urban medical insurance system and its prospects are not good.

\section{Underlying Reasons}

Several factors contributed to the problems with China's health care system. Insurance system reform is inevitably influenced by what is happening elsewhere in the health sector and the social security sector. There are also technical problems, but socioeconomic and cultural problems played a more important role. Several of these can be identified.

\section{Attempted Reforms since the Early 1980s}

Since the early 1980s, China has been making changes in its economic and administrative systems. This has changed China's socioeconomic scenario dramatically and heavily affected China's health system. Private initiatives and market forces have largely supplanted government planning, and the government dramatically changed the way it financed health care. The central government's investment in health care services was dramatically reduced. Between 1978 and 1993, the government's share of total national expenditure on health (exclusive of GIS spending) fell from 28 percent to 14 percent and the allocation by rural cooperative medical plans fell from 20 percent to 2 percent (20). The available public funds for health care only covered basic personnel wages and new capital investments, which were about $25-30 \%$ of hospital expenditures; instead, the government gave hospitals and health centers a large degree of financial independence. Bonus payments had to be funded from the hospital's earned profits, thereby allowing the private market to play a role in the health sector. At the same time, the central government transferred much of the responsibility for funding health care services to provincial and local authorities and required them to provide that support through local taxation [11]. Meanwhile China decentralized its public health system, and reduced central governmental funding for local public health efforts [12]. The government also allowed private ownership of health facilities and private clinical practices. Private investment in new hospitals was promoted by allowing private facilities to charge higher fees than those allowed for public hospitals. 


\section{Technical Factors}

Price controls. To ensure access to basic care, the government continued tight price controls over routine diagnostic tests, treatments and pharmaceuticals in publicly owned hospitals and clinics. However, it permitted facilities to earn profits from new drugs, new tests, and technology, with profit margins of 15 percent or more. Thus the price controls did not fully limit the cost of health care within the system.

Compensation mechanism. The government modified its salary-based system of compensating hospital physicians to include bonuses determined by the revenue the physicians generated for their hospitals. The major source of those revenues came from sales of profitable new drugs and technologies. The result was an explosion in sales of expensive pharmaceuticals and high-tech services, such as imaging, and rapid overall increases in health care prices and spending [13]

Payment method. A fee-for-service method was dominant in most hospitals and health settings, an approach that lacked cost-containment measures on the supply side. Once again, this approach inevitably stimulated the physician to rely on excessive (even unnecessary) drug prescriptions and tests on high-tech medical equipment to generate higher hospital revenues.

Administrative capacity. This problem arose due to the lack of guaranteed enrollment and premium collection. Cities and counties (the budgetary units) do not have the legal authority to require participation, since China does not have a social insurance law. Local governments are reluctant to implement such a program for fear of running up budget deficits. Any deficits of the insurance funds must be absorbed by the local governments, which may represent a drain on their budgets [14]. Thus, as a limited sector reform, health reform measures in China still lack support from other socioeconomic policies, especially in the health sector price, organizational function, and payment methods sphere. In fact, a successful strategy to contain medical cost inflation heavily depends on payment reform. The government, as well as many localities, does not have the administrative capacity and technical knowhow to ascertain and adopt appropriate payment methods.

Benefit reduction. Compared to the old system of using the GIS and LIS, the concept of "free medical care" no longer exists. The premium contribution based on the $8 \%$ of the current wage bill can only cover about 70 percent of the total outlay under the old systems of GIS and LIS [15]. The new system has a ceiling on coverage of individual medical expenditures (equivalent to four times the average wage in the region). Imposition of this ceiling is due to budget constraints and a political emphasis on 'wide coverage', i.e., benefiting most of the enrollees. In addition, the dependents of the urban workers, who used to receive partial coverage, are now not covered. These facts mean that the new system may represent a benefit reduction for enrollees, one of the major reasons for the public dissatisfaction with the new system.

Exclusion of catastrophic diseases. The current plan has weak fund resistance against catastrophic illnesses due to its ceiling of four times the average wage of the workers. Only government employees are entitled to a supplementary insurance plan. State-owned and private enterprise employers may buy commercial insurance to cover their employees' catastrophic illnesses, or employees may buy it for themselves. Still, either way is voluntary rather than mandatory. This approach leaves a substantial number of urban employees uncovered when they incur catastrophic diseases.

\section{Socioeconomic and Cultural Factors}

Public and privately owned economic composition. One of the major points for arguing that China's health system has been "basically unsuccessful" is that the "business- and market-orientation" of the medical and health system is in conflict with its goal of public health. According to China's official statistical abstract for the gross industrial output for the nation in 1998, state-owned industry accounted for $28.5 \%$ of the total output, collective industries accounted for $38.3 \%$, individual companies accounted for $18.9 \%$, and other types rounded out the list with $19.9 \%$ of the total output. Thus the ratio 
between the public vs. privately owned output is roughly in the proportion of $60 \%$ vs. $40 \%$. In addition, in terms of fixed assets investment, state-owned economic investment represented 55.0\%, collective economy $13.1 \%$, and individual investments $12.8 \%$. Thus the public vs. private proportion of total economic investment was $68 \%$ vs. $13 \%$. That means public companies account for the majority of economic production in China, but privately owned companies also contribute a substantial proportion for the society's total economy. The health system is not a simple reflection of this economic framework, but these facts and other statistics serve as a key reference to address the fundamental question of whether the health system reform should introduce business- and market-orientation, and to what extent. In current practice, market orientation has exceeded its original planned domain and played a major role that allows providers to overuse medical service and drugs for high or even exorbitant profit. This situation greatly deviates from the initial intent and penalizes consumers and the society.

Societal population and demography -- urban population facts and health trend

The current insurance system only covers urban employees, but leaves a wide gap between them and the uninsured urban population. By the end of 2002, there were 660 cities in China with a total urban population of 353,437.8 thousand people [16]. Urban unemployment population was 5.95 million, equivalent to an unemployment rate of $3.1 \%$. If laid-off worker and other unregistered populations are included, the actual figure is estimated to rise to 30 million and 12 to $15 \%$ [17]. Urban poor and financially vulnerable populations total 14.6 million, with a rate of 4.1 percent [18]. Moreover, there are 46-to-100 million rural migrants in China's cities [19] who are not covered by this insurance system because they don't have a formal urban household registration. Although there would be some overlap among the above populations, these figures provide a basic picture of China's urban situations. In 2000, $6.95 \%$ of China's population was age 65 or older [20], which signaled that China had become an aging society. In Shanghai, for example, the urban aging population was $13 \%$ of its total population in 2001 [21]. Similarly, in any other of China's metropolitan areas, the urban vulnerable populations account for substantial proportion of the total population. This fact cannot be ignored and needs special attention from the society. The aging population is closely related to chronic diseases and costly expense, which has put a demanding challenge on the insurance system. These subpopulations jointly indicate the weakness and relatively poor performance of the current "basic" benefit design, which either excludes the vulnerable population or offers the aging population a weak benefit against catastrophic medical expenses due to financial constraints. Health insurance, as one indispensable part of the social protection system, should take them into consideration in its benefit design.

Variations in urban socioeconomic development. There is also great disparity in the cities' socioeconomic development. In 2005 gross regional product per capita in Suzhou amounted to 66766 Yuan. It was 51474 Yuan in Shanghai, and 60801 Yuan in, Shenzhen 60801. The lowest one was 2394 Yuan. Thus the ratio of the two extremities was 27.8 times. Urban family per capita income amounted to 27596 Yuan in Shenzhen, 16683 Yuan in Shanghai, and 15638 Yuan in Beijing; the lowest ones only ranged between 4000-5000 Yuan, with a gap of 5-to-6 times [22]. These facts imply that a diverse need and demand for health insurance must be taken into considerations to reflect significant regional socioeconomic variations. In particular, the national standard 'basic' benefit package should be carefully designed to represent equity and aim to allieviate poverty. At a minimum, a basic benefit package in the insurance plan should meet the bottom line for national coverage and leave localities some autonomy.

\section{Cultural reasons}

Government understanding of health care. As the cost problem has become obtrusive, health care is no longer seen as welfare entitled by everyone. Instead it becomes an individual responsibility. This leads to a benefit reduction for employees and thus represents public dissatisfaction.

Traditional culture and belief. Egalitarianism is a commonly accepted social norm, which peaked in 
Mao's era in 1950s. The current system created great access disparity in urban populations by its formidable price, one of the major criticisms from State Council's report in 2005. It has deviated substantially from the nation's social norm and its basic function of serving the public.

A second cultural factor is that of dependency. Dependency on employers is another important psychological Chinese characteristic. People are used to relying on the government or employers for housing, retirement and health care, or even dependents' employment. GISs and LISs had provided unlimited health care coverage. Now the current insurance benefit is only partial and limited, and focused on self responsibility for health and medical care. Especially when the meager given benefit resource was depleted, people often had to pay a considerable amount out of pocket for health services. The government and employers no longer served a role as the last resort for full protection. Breaking away from tradition, along with benefit reduction, always makes people frustrated and distressed.

A third cultural factor is that of mediocrity and conservative beliefs, which has long been a deeply rooted tradition in Chinese mind sets. When a new event occurs, people usually wait and watch because they are uncertain of the potential risk. Even if the status quo is not good, they don't want to be the first one to accept or participate in a new system. They wait to act until other people have tried the system and demonstrated that it has little or no risk and greater benefits. This is the case for many employers' attitude toward participation, because currently urban employee health insurance is not compulsory (which, in turn, determined the magnitude for social risk pooling funds). In fact they were far from sufficient and have a weak protective function in either urban enrollee coverage or benefit coverage.

A fourth cultural factor is indifference and adverse selection. Economic evolvement has changed people's moral views from altruism to the other side, at least to a degree of indifference to others' interests. They only care for their own interests and don't want to spend money on others. That is why many employers did not participate in the insurance system if their workforce was mostly younger individuals. The new social insurance system left the burden of catastrophic illnesses to commercial insurance, but it was voluntary. Younger employees, facing a small risk, have little or no incentive to purchase commercial insurance.

A fifth cultural factor is that it created a moral hazard. Urban enrollees entitled to insurance benefits tend to take care of their family members and friends who were excluded from the insurance or could not afford medical bills. The result was over utilization of their own benefits in the form of excessive or expensive medical service tests and drugs. This led to a considerable waste of social health resources and partly contributed to cost escalation.

A sixth factor is confidence and trust in the government. Each succession of government sought to improve their own record, sometimes without considering what they would leave for their followers. However, the people suffered losses in health benefits due to frequent discontinuity and inconsistence of government policies. As a result, they became indifferent to the current system.

\section{Proposals for future reform}

Reform principle and positioning. A major goal of the health system is equity and reducing poverty, which places the emphasis on who benefits from the insurance program, arguing that it should differentially be the bottom line--the poor and financially vulnerable, not necessarily those who already enjoy the "basic" health care for better service. Equity does not necessarily mean to provide "equal" health service to every enrollee. Meanwhile, governments should consider socioeconomic disparities among cities and balance the needs and demands of different social groups and localities if a national standard "basic" benefit package is assumed.

Next, properly dealing with the role of "business- and market-orientation" mechanism in the health system is closely associated with solutions to roaring cost and access disparity. The national economic composition has dictated the role that market mechanism play in the health system. Market mechanisms reflect people's willingness and ability to pay and can lead to disparate access and quality. 
Thus the government should play a major role to achieve the goal of equity and poverty alleviation. As a strategic plan the health system should comprise both the government regulation and marketorientation mechanism, and stand somewhere between them. The latter should serve as a supplemental one, but not a major one, so one is not easily confused by the debate over which model should persist and whether a proposed policy should totally veto current practice and shift from one extremity to the other.

Another strategic consideration is the benefit design, based on urban population's characteristics and health trends. It should combine both basic and comprehensive packages, strengthening the defense function against catastrophic diseases.

Policy recommendations. Based on previous analysis, it should be highlighted that each nation has its own socioeconomic, political and cultural characteristics that need to be integrated into the health system. Thus any proposed solutions should match urban situations as possible as they can. We suggest:

(1) Constrain market mechanisms in the health system to a reasonable sphere. Namely it should play a partial and supplemental role, while government regulation should expand to a dominant position. In a joint effort to contain formidable cost, reduce prices in drug and medical equipment tests and increase prices for medical services with an incentive in recognition of medical technology value. Another option is to experiment with the payment method by transferring fee-for-service to capitation. Reform related to hospitals and physicians payments can be addressed by introducing or referring those based on DRG and CPT method, removing physicians' bonus and increasing their fixed salaries to an acceptable level. As a powerful support, the government should focus on prevention and public health interventions to maintain the basic level of public health status, which proved to be cost-effective.

(2) There is also a need to identify high-risk vulnerable urban populations, including the unemployed, laid-off workers, urban poor and financially vulnerable population, rural migrants, and aging population. Policy-makers need to learn from other developed nations' models on how to provide sufficient healthcare assistance to vulnerable groups and deal with disparity among cities. According to the nature of the population, the government should design different schemes for different social groups, and provide special schemes for special groups at a high risk of ill health, for example, design a new insurance scheme that deductible varies according to income and exempt low-income groups from paying premiums and deductibles [23].

(3) Implement mandatory social insurance system in wealthy pilot cities to ensure adequate social risk pooling funds against catastrophic diseases. At the same time other approaches of financing are also recommended. Governments committed to equity could collect revenue on the basis of ability to pay along with some government financial support and disburse it on the basis of need, especially for the urban poor and vulnerable groups [24]. Donations from enterprises or social groups should be encouraged and motivated.

(4) Ensure each plan does not have major variations within each subpopulation with similar characteristics. To reduce perceptions of unfairness, the government and employers should provide more psychological care and support to their employees and vulnerable urban groups, if insurance benefits are not available, in an aim to maintain people's dependency expectation as well as their confidence and trust to government.

\section{Conclusion}

Just as an economic base dictates superstructure and productivity dictates production relationships, so does society and culture dictate a nation's policies and institutions. Health policy and system is by no means an exception. No health system can survive without its deeply rooted social environment and cultural context. That is why, even if a health system is technically mature and feasible, it still might not work in a certain context, and why one nation should be cautious not to simply transplant a 
successful model from another country to its own. There is no perfect health plan model suitable for every population and nation, but only appropriate ones for specific nations and specific populations at the right place and right time.

The healthcare system framework for urban employees in China was established in the early 1950s. The first stage (1950s-1980) mainly consisted of two parts, GIS and LIS, and they achieved remarkable success with limited resources, contributed to economic development and social stability, and won WHO's high praise and was set up as a paradigm. The simply reason for its success lay in its suitability to China's economic, political and societal situation at that time: ideology congruency, operation feasibility, strategy appropriateness, and delivery and organization appropriateness.

In the first half of second stage (1980s-1991), major reform measures consisted of the introduction of demand-side and supply-side cost sharing in response to cost escalation brought on by reliance on the market economy. These measures played a role in mitigating China's rapid health care cost inflation, thus relieving some of the financial pressure on enterprises. However, these reforms were not complete and left many fundamental problems unsolved. The radical transition of the system eradicated the successful experience and tradition of the previous stage. In the second half of this stage, beginning in 1992, the focus of reform shifted to the more fundamental problems-expanding workers' risk to the city level along with the original goal of cost containment. A new approach was adopted: a combination of medical saving accounts and a social risk pooling fund, based on models in Singapore and Germany. This new system expanded coverage to private sector employees and provided a more stable source of financing with its risk pool at the city level. Meanwhile it brought about new problems as well as exacerbated some original ones. These included cost escalation, disparity of access to health service, and excluded a large portion of uninsured, unemployed, poor and vulnerable urban population. The reasons are complex, including both technical flaws in design and health plan incongruence with current China urban socioeconomic and cultural situations. In details, the scheme excluded a substantial portion of urban poor and vulnerable population and didn't reflect the nation's social aging trend. Its benefit package only had a weak resistance to catastrophic diseases expenses due to inadequate social risk pooling fund. The system only partly matched property ownership. Market mechanisms played a major role in the system, resulting in a formidable cost differentiation of people on the basis of willingness and ability to pay. Reforms from related sectors were either unmatched (e.g., price) or absent (e.g., payment methods, compensation mechanism. The insurance system's operation and performance had significant conflicts with social norm and culture.

It should be noted that health care systems never work alone, and more importantly there is not a best model for every nation or any nation all the time, only a most suitable one for unique society and unique population in the unique period. Future reform directions should explore most suitable model for current urban situations. Future health insurance reform should consider a goal of equity and reducing poverty rather than providing better benefit for existing beneficiaries. Market mechanism should give way to government regulation. Specific recommendations include constraining market mechanism in the health system to a reasonable sphere, expanding the government's role to a dominant position, restoring and enhancing prevention and public health intervention functions; designing different schemes for different social groups, especially those at high risk of ill health; implementing mandatory social insurance system in wealthy pilot cities to ensure adequate social risk pooling funds, collecting revenue on the basis of ability to pay along with some government financial support and disbursing it on the basis of need, encouraging and motivating donations from enterprises and social groups, and reducing benefit inequality to alleviate their perception of unfairness. The government and employers should provide more psychological care and support to their employees and vulnerable urban groups if insurance benefits are not available, in an aim to maintain expectations, confidence and trust in government. 


\section{References}

[1] D..Blumenthal, W. Hsiao, Privatization and its discontents: The evolving Chinese health care system. The New England Journal of Medicine, 353:11 (2005), 1165-1170.

[2] Kristof, N. D. Kristof, Chinese grow healthier from cradle to grave. New York Times, April 14, 1991.

[3] T. Hesketh, W. X. Zhu, W. Xing Health in China: From Mao to market reform. British Journal of Medicine, 314 (1997), 1543-1545.

[4] Y. Liu, Reforming China's urban health insurance System. Health Policy, 60 (2002), 133-150.

[5] T. Hesketh, W. Zhu, Health in China: The healthcare market. British Medical Journal, 314 (2004), 1616-1618.

[6] Liu, (2002)

[7] Liu, (2002)

[8] Liu, (2002)

[9] Liu, (2002)

[9] Lliu, (2002).

[11] Hesketh, Zhu (2004).

[12] Y. Liu Y. China's public health-care system: Facing the challenges. Bulletin of the World Health Organization, 82 (2004), 532-538

[13] Blumenthal, Hsiao, (2005).

[14] Liu (2002).

[15] Liu, (2002).

[16] City construction statistic bulletin in 2002

[17] Asian bank panel. China urban poverty study. 2000

[18] Liaowang News Periodical. Focusing on 14 million urban poor population 2002

[19] China Market Economy Newspaper 2001, People Political Consultation Newspaper 2007

[20] China Population Web 2004

[21] Shanghai Municipal Statistics Bureau 2001

[22] China Urban Economy Web. Stage Characteristics and Locality Variations in City's Modernization in China 2007

[23] Weizhen Dong. Health Care Financing Reforms in Transitional Society: A Shanghai Experience. J Health Popul Nutr, 21:3 (2003), 223-234

[24] Weizhen Dong. Health Care Financing Reforms in Transitional Society: A Shanghai Experience. J Health Popul Nutr, 21:3 (2003), 223-234 